\title{
Automated Ultrasound Doppler Angle Estimation Using the Probabilistic Hough Transform
}

\author{
Yong Chen $^{1}$ \\ ${ }^{1}$ College of Software, East China JiaoTong University, Nanchang, JiangXi, China \\ chenyong0706@163.com
}

\begin{abstract}
Modern Doppler ultrasound can estimate the velocity of blood flow in vessels quantitatively and noninvasively. Once the Doppler angle, i.e. the angle between the insonating beam and the direction of blood flow, is given, the velocity of flow can be estimated using the standard Doppler equation. However, the operator is required to manually tune the vessel slope cursor based on the direction of the vessel wall in the Bmode image to specify the Doppler angle. This manual tuning process is time consuming and error prone. In this paper, a novel automatic way of specifying the Doppler angle is proposed to improve the efficiency and accuracy of the blood flow velocity measurement. Our approach starts with the image preprocessing and segmentation of blood vessel in ultrasound common carotid artery image. The segmentation step is followed by an estimation of Doppler angle based on the probabilistic Hough Transform technique. On 15 ultrasound video sequences of the common carotid artery taken from 4 healthy subjects, the results generated by the proposed method are compared to the angles specified by an expert operator. The deviation of the angles estimated by the proposed method from the angles specified by the operator is 4.15 degrees \pm 2.92 (mean \pm standard deviation). The proposed method eliminates the need of manually tuning of the vessel slope cursor and the estimated angles are in highly agreement with the ones specified by the operator. It has the potential to enhance the work flow and improve the speed of the ultrasound spectral Doppler examination.
\end{abstract}

Keywords: Doppler angle estimation; image segmentation; common carotid artery; ultrasound

\section{Introduction}

The distribution of the blood velocities inside a vessel provides valuable clinical information to the clinicians. For example, abnormal blood flow velocity indicates that one of the blood vessels maybe blocked or broke open. One of the advantages of Doppler ultrasound is that it can measure the blood flow velocity noninvasively and quantitatively. Once the Doppler angle, i.e., the angle between the insonating beam and the vessel slope is given, the blood flow velocity $v$ is calculated by the Doppler equation as follows:

$$
v=\frac{f_{d} \times c}{2 \times f_{0} \times \cos \theta}
$$

where $\mathrm{c}$ is the speed of ultrasound wave in the tissue, $f_{i}$ and $f_{0}$ are the Doppler frequency and center frequency of the transducer respectively, and $\theta$ is the angle between the insonating beam and the direction of blood flow.

Figure 1 shows a screen shot of spectral Doppler which is available on most commercial ultrasound medical imaging system. In the B-mode image, the longest line indicates the direction of insonating beam and the two short parallel line segments indicate the time-gated window. The ultrasound signals being used to estimate the 
velocity are sampled inside this gated window. The cursor in the middle of the gate indicates the direction of blood flow.



Figure 1. A Screenshot of Spectral Dopple

When an operator is going to use the spectral Doppler function, he first uses trackball or mouse to place a gate, e.g. $1.5 \mathrm{~mm}$ wide, along the direction of insonating beam in the middle of the vessel. Then he needs to manually tune the cursor, e.g., via a toggle switch, to make it align with the vessel slope. Once the gate and cursor are given by the operator, they will be used to estimate blood flow velocity according to equation (1). Here comes the problem. If the angle step size is preset as relatively coarser, then there are relative fewer preset angles and the process is susceptible to error; on the other hand, if the angle step size is preset as relatively finer, the operator may need to spend more time and effort to reach the desired angle.

To address this dilemma, several methods have been proposed. Saad et al. [1] proposed to use a computer vision approach to estimate the Doppler angle automatically. Their method can be roughly divided into four steps. In the first step, the information of color Doppler image was being used to binarize the image. But the authors also pointed out that the resultant binary image often suffered from a well-known artifact of the color Doppler imaging which is called "color bleeding". For the sake of preventing "color bleeding", they used shape decomposition technique to decompose the binary image into several individual vessels in the second step. In the third step, they chose to use skeleton representation technique to extract the direction of the segmented vessels. Then at last step, the Doppler angle was generated by applying a least square line to the skeleton points around the given point. In [2], LiHong Pan et al. applied a local search based on a combination of intensity-only and intensity-difference thresholds to locate the edge points along the vessel slope, which in turn were used to estimate the vessel slope. In [3], Antoine Goujon proposed to use the isotropic tracing of rays over the region of interest and the thresholding of local histogram to detect the edge points along the vessel slope. These edge points were later fitted to determine the Doppler angle. In [4], Criton and Routh suggested to utilize duplex ultrasound beams passing through the Doppler gate to determine the direction of blood flow.

In this paper, we have suggested a novel automated Doppler angle estimation method, which is called DAEM. DAEM assumes that the direction of blood flow is parallel to the slope of the vessel wall. So once the slope the vessel wall is determined, the Doppler angle can be determined too. As shown in Figure 2, after the operator manually placed a gate inside the vessel, DAEM will do the following computational steps: image preprocessing with VMF filter [5], edge points detection by Otsu's algorithm [6]; reduction of edge points; application of the probabilistic Hough Transform (PHT) [7] technique to find the direction of the blood flow. PHT is very good at detecting straight line, which is in turn used to approximate the wall-lumen boundary in longitudinal section. 
As far as we known, similar approach has not been addressed in any of the previous studies. To validate the results of DAEM, the results were compared with angles manually set by an expert.



Figure 2. The Flow Chart of DAEM

\section{Main Principle of Standard Hough Transform and Probability Hough Transform}

The objective of standard Hough Transform (SHT) is to find all straight lines in an image that passed through a number of continuous points. Duda and Hart [8] stated that any straight line, e.g. $\mathrm{y}=\mathrm{mx}+\mathrm{b}$, in the image domain can be equally represented by polar representation:

$$
r=x \cos \theta+y \sin \theta
$$

where the pixel $(\mathrm{x}, \mathrm{y})$ lies on a straight line, $\mathrm{r}$ is the distance from the origin to this straight line, and $\theta$ is its angle with the x-axis. If we establish another coordinate, called Hough Domain, with $\theta$ the $\mathrm{x}$-axis and $\mathrm{r}$ the $\mathrm{y}$-axis, it can be observed that a straight line in the image domain is essentially a point in the Hough Domain and a sinusoid curve in the Hough Domain is corresponding to a point in the image domain. Then the location in the Hough Domain where two sinusoid curves met, is corresponding to a straight line that pass through the two corresponding points in the image domain. SHT makes use of this property to convert the complex problem of detection of straight lines in the image domain into a simpler problem of detecting local-peaks in the Hough Domain. It has been applied to a wide variety of medical image applications since its publication. For example, Golemati et al. [9] successfully applied SHT to segment the longitudinal and transverse sections of the carotid artery.

But there are some problems with SHT. One disadvantage of SHT is that a pixel is counted many times when several lines pass through it, which may result in the detection of false lines. Another disadvantage is that it doesn't provide information of line segments. PHT [7] adds a few modifications to overcome these disadvantages. It not only reduces the number of lines greatly by removing the edge points along the unqualified lines, but also provides the information of line segments.

Figure 3 shows an example of the comparison of SHT and PHT. The original ultrasound image is shown in Figure 3a. Then the original image is binarized using Canny operator. The results of lines detection for the binary version of original images using SHT and PHT are shown in Figure 3b and Figure 3c respectively. 




Figure 3(a). The Original Ultrasound Image



Figure 3(b). The Result of Lines Detection Using the SHT (The White Represent the Edge Points And the Red Indicates the Detected Lines)



Figure 3(c). The Result of Lines Detection Using the PHT (The White Represent the Edge Points and the Red Indicate the Detected Lines)

Figure. 3 the comparison between the SHT and the PHT: (3a) the original image; (3b) the resulting lines detected by the SHT; (3c) the resulting lines detected by the PHT.

\section{DAEM}

In this section, we describe the DAEM that is conceptually made of following cascaded steps: the specification of ROI, image preprocessing with VMF filter, edge points detection using Otsu's algorithm, reduction of edge points, application of PHT, detection of dominant line. 
First, the operator need to manually place a gate in the middle of the vessel of common carotid artery (CCA). Let's denote the center of the gate as (cx, cy). Now it is time to determine the ROI, a rectangular area containing the posterior wall of the vessel. After applying the knowledge that the average diameter of the CCA for adult males and females is $6.52 \mathrm{~mm}$ and $5.11 \mathrm{~mm}$ respectively [10], we regulate the ROI should be of $6.5 \mathrm{~mm}$ height and $16 \mathrm{~mm}$ width, and the left-top location of the ROI should be (cx $-8 \mathrm{~mm}$, cy). The following computations would focus on the extracted ROI. This reduction of image area not only saves the computational cost, but also eliminates the possibility to detect unwanted structures. An example of ROI specification is shown in Figure 4.



Figure 4. An Example of ROI Specification

Since ultrasound speckle often degrades the quality of the ultrasound image and makes segmentation difficult, it is better to do some image preprocessing to reduce the speckle noise before segmentation. It is desirable to decreasing speckle and preserving valuable information, such as boundary of the CCA, at the same time. In this work, the vector median filtering (VMF) [5] has been used to do speckle reduction in the extracted ROI area. The VMF is a nonlinear filtering strategy which is good at removing impulse and multiplicative noise, making it very useful to remove speckle. For a pixel centered at its $\mathrm{n} \times \mathrm{n}$ neighborhood, the VMF replaces its intensity value with that of the pixel that has the minimum summation of Euclidean distance regarding to all other pixels in the neighborhood. As for our case, a $3 \times 3$ neighborhood is employed. An example of the filtered ROI is shown in Figure 5a.

Then the adaptive thresholding Otsu's algorithm is applied to the filtered ROI to detect the edge points between the two main image classes, i.e., the blood and the arterial tissues. The threshold is automatically detected by maximizing the between-class variance. The resulting binary image shows $1 \mathrm{~s}$ at the edge locations and 0s at locations elsewhere. Dana E. Ilea etc. [11] have shown that the Otsu's algorithm excel at finding the border points of the posterior wall of the CCA. An example of the detection of edge points using Otsu's algorithm is shown in Figure 5b.

Because we are interested in making use of edge points which are neighborhood to the vessel to estimate the slope of the vessel, we propose to reduce the number of edge points to remove the useless edge points. For every column, only the three top edge points are kept and others are discarded. One example of the reduction of edge points is shown in Figure 5c.

The last step is to apply the PHT. It often returns more than one lines. We simply choose the line with the maximum length as the dominant line. One example of the resulting dominant line is shown in Figure 5d. The intercept of the dominant line is calculated to represent the direction of the blood flow. Then the Doppler angle can be determined based on the insonating beam and the direction of the blood flow, which in turn leads to the determination of the velocity of blood flow according to equation (1). 




Figure 5(a)



Figure 5(c)



Figure 5(b)

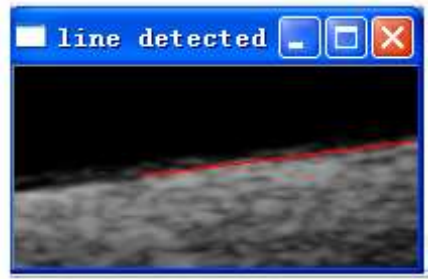

Figure 5(d)

Figure. 5 an illustration of the process of the DAEM: (5a) an example of VMF filtered ROI; (5b) an example of the detection of edge points using Otsu's algorithm; (5c) an example of the reduced edge points; (5d) an example of the detected line.

\section{Results}

Four healthy volunteers aged 25-39 with no history of cardiovascular disease were involved in this experiment. The common carotid arteries of the right side of their necks, 2-3 centimeters proximal to the bifurcation, were scanned by an expert. A total of 15 ultrasound image sequences with 130 images were obtained using Saset iMago C21 system (SASET Healthcare, San Francisco, CA) with a $10 \mathrm{MHz}$ linear transducer. The capturing resolution of the images was $0.07 \times 0.07 \mathrm{~mm}^{2}$. The sonographic setting remained unchanged throughout the process of data acquisition.

To test the robustness of the DAEM, we paid attention to include ultrasound images with different angles of vessel slope. Figure 6 shows the result of the DAEM when it was applied to three of these images.



Figure 6(a). The Blue Represent the ROI and the Red Indicate the Detected Vessel Slope 




Figure 6(b). The Blue Represent the ROI and the Red Indicate the Detected Vessel Slope



Figure 6(c). The Blue Represent the ROI And the Red Indicate the Detected Vessel Slope

Figure.6 An illustration of the robustness of tahe DAEM when it is applied to images with different angles of vessel slope.



Figure 7. The Comparison of the Angle Line Estimated By the DAEM (The Dashed Green) With That Set by the Expert (The Solid Red Line)

To verify the accuracy of the DAEM, we compared the results generated by the DAEM with the manual angles set by the expert sonographer. We regard the manual-set angle lines as the ground truth (GT). So the closer the angle lines estimated by the DAEM are to the GT, the better performance the DAEM achieves. Figure 7 shows an example of the comparison result. The solid red line is the angle 
line set by the expert to represent the vessel's orientation at this site and the dashed green line represents the angle line estimated by the DAEM.

The measurement of the overall deviation errors from the ground truth data is shown in Figure 8. It uses the boxplot() function from Matlab to depict the overall deviation errors. The deviation of the angles estimated by the DAEM from the ground truth data is 4.15 degrees \pm 2.92 (mean \pm standard deviation).



Figure 8. The Deviation Error of the DAEM

Implemented in $\mathrm{C}$ language, the computation time required to calculate the Doppler angle is about 0.04 second in a desktop computer with $2 \mathrm{G}$ memory and Intel Celeron 2.5 $\mathrm{GHz}$ CPU inside.

\section{Discussion}

Spectral Doppler technique has long been used to quantitatively measuring blood flow velocities, and these measurements provide valuable information for the diagnosis and grading of arterial stenosis. However, one drawback of Doppler ultrasound is that the operator has to manually adjust the Doppler angle. This manual approach suffered from two limitations. The first limitation is that the inter-operators and intra-operator variance. The second is that manual adjustment is labor-intensive and time-consuming. The method reported here is fully automated and real-time, which has important clinical meanings. It frees the operator from labor-intensive adjustment of the cursor and also eliminates the possibility of inter-operators and intra-operator variance occurred in manual approach. Meanwhile, the result returned by the DAEM shows highly agreement with the ground truth data. So the proposed method can be considered as a valid alternative to the manual approach.

The innovative aspects of the DAEM includes: a) the application of the probabilistic Hough Transformation to estimate the Doppler angle; b) the reduction of edge points to enhance the accuracy and speed of computation.

As can be observed from the previous methods $[1,2,3]$, the technique of least squares line fitting of the edge points was frequently used to estimate the Doppler angle. Though this technique works reasonably well in most cases, its performance degrades severely when the adopted edge points would produce large fitting error [1]. But if the probabilistic Hough transform is used instead, the case described above can be avoided because the edge points having large fitting error can't be in the same straight line. So the PHT is immune to these kinds of edge points which means that the PHT would achieve better performance than the technique of least squares line fitting. 
Another novelty of this method is the reduction of edge points. If we apply the probabilistic Hough transform to the detected edge points directly after the application of adaptive thresholding Otsu's algorithm, then it would produce many more lines than the lines produced by our method. The worse is that some lines may even larger than the length of 'dominant' line as described in section 3, which would result in erroneous estimation of Doppler angle. And the experiments proved that our method of reduction of edge points solved this problem gracefully.

\section{Conclusions}

In this work, we have proposed a novel technique involves several integrated processing steps aiming to automatically estimate the Doppler angle. The initial result with the obtained 130 images was very encouraging and the method has been integrated into a Saset iMago C21 system (SASET Healthcare, San Francisco, CA). We have performed a quantitative comparison of the accuracy of the DAEM vs. the Doppler angles manually set by expert. The result showed that they were in highly agreement. So the DAEM has the potential to be an alternative for the labor-intensive manual adjustments of the Doppler angle.

The future work will be focused on evaluating our method under controlled clinical trials, especially those patients who are suffered from advanced cardiovascular conditions, such as those with severe stenotic cases.

\section{References}

[1] A. A. Saad, T. Loupas and L. G. Shapiro, "Computer Vision Approach for Ultrasound Doppler Angle Estimation”, Journal of Digital Imaging, vol. 22, no. 6, (2009), pp.681-688.

[2] L. H. Pan, M. J. Washburn and L. Y. L. Mo, "Method and Apparatus for Automatic Doppler Angle Estimation in Ultrasound Imaging", U.S. Patent 6,068,598, (2000).

[3] A. Goujon, "Method for Automatic Measurement of the Doppler Angle and Arrangement for Carrying out the Method", U.S. Patent 5,690,116, (1997).

[4] A. Laure Criton and H. Frances Routh, "Automatic Flow Angle Correction by Ultrasound Vector”, U.S. Patent 6,464,637 B1, (2002).

[5] J. Astola, P. Haavisto and Y. Neuvo, "Vector Median Filters", proceedings of the IEEE, vol. 79, no. 4, (1990).

[6] N. Otsu, "A Threshold Selection Method from Grey-level Histograms", IEEE Transaction on System, Man and Cybernetics, vol. 9, no. 1, (1979), pp.62-66.

[7] C. Galambos, J. Kittler and J. Matas, "Gradient-based Progressive Probabilistic Hough Transform”, IEE Vision Image and Signal Processing, vol. 148, no. 3, (2002), pp. 158-165.

[8] R.O. Duda and E. Hart, "Use of the Hough transform to detect lines and curves in pictures", Communications of ACM, vol. 15, (1972), pp. 11-15.

[9] S. Golemeati, J. Stoitsis, E. G. Sifa-kis, T.Balkizas and K. S. Nikita, "Using the Hough transform to segment ultrasound images of longitudinal and transverse sections of the carotid artery", Ultrasound in Med. \& Biol., vol. 33, no. 112, (2007), pp. 1918-1932

[10] J. Krejza, M. Arkuszewski, S.E. Kasner, J. Weigele, Ustymowicz, R.W. Hurst, B.L. Cucchiara and .SR. Messe, "Carotid Artery Diameter in Men and Women and the Relation to Body and Neck Size", Stroke, vol. 37, (2006), pp. 1103-1105.

[11] D. E. Ilea, C. Duffy, L. Kavanagh, A. Stanton and P. F. Whelan, "Fully Automated Segmentation and Tracking of the Intima Media Thickness in Ultrasound Video Sequences of the Common Carotid Artery", IEEE Transactions on Ultrasonics, Ferroelectrics, and Frequency Control, vol. 60, no. 1, (2013). 


\section{Author}



Yong Chen, he got his ph.D. degree in SiChuan University in 2014. His ph.D. work focus on the processing of medical ultrasound signal and images. He is now a lecturer in College of Software, East China JiaoTong University. His research interests include medical image processing and code clone detection. 